\title{
Patient experience of flap monitoring following free flap surgery for oral cancer reconstruction
}

\begin{abstract}
Regular free flap monitoring is an essential component of the post-operative management of patients following microvascular free tissue transfer (FTT). The frequency and duration of observation varies between units and there is no nationally agreed consensus. Best practice can be informed by patient feedback; however to patient perspectives of flap monitoring are absent from the literature. The aim of this study was to perform a cross-sectional survey of a consecutive group of patients following FTT to ascertain their experience of monitoring. A study specific questionnaire was used following consultation with the patient and carer forum. In the unit there were 150 patients treated by TFT in 2015 and 2016, of which 71\% (106) responded to the survey. One quarter (28\%) wanted more information about flap monitoring. Patients were generally not worried that much by the flap observations (86\%); however one-third (32\%) reported substantially disturbed sleep. Just over half (53\%) were notably relieved when monitoring changed from hourly to every four hours, and almost all (98\%) expressed a lack of worry about this changeover. Nearly half (45\%) would have preferred less frequent observations whilst asleep. In conclusion, this perception could help inform a patient information leaflet on flap monitoring. Consideration could be given to reducing the frequency of monitoring, such as from $12-6$ am on night two the check at 1,3 and 5 am could be omitted. This minor change could make a substantial difference for the patient but the impact of this on salvage needs to be audited.
\end{abstract}

\section{Keywords}


Quality of life, free flap surgery, oral cancer, flap monitoring, free tissue transfer

\section{Introduction}

Postoperative monitoring of microsurgical free-tissue transfers for head and neck reconstruction forms an essential part of post-operative care of head and neck cancer. ${ }^{1,2}$ Early detection of compromise is very important as early intervention is associated with improved flap salvage rates and overall success.$^{3}$ Flap compromise tends to occur in the first 72 hours after surgery and intensive monitoring is carried out during this period. ${ }^{3,4}$ The protocol for free flap monitoring varies between units and there is no clear consensus regarding best practice. The onus on flap monitoring also varies between nursing staff and junior doctors. ${ }^{5}$ Although all units used clinical examination to assess viability of the flap, just over half (57\%) had a written protocol for free flap monitoring. ${ }^{6}$ Clinical examination is the mainstay of monitoring in the 60 Oral and Maxillofacial units across the UK and assessment includes skin texture, colour, temperature and capillary refill. ${ }^{6}$ The frequency of observation varies between 15 minutes and 8 hours with the interval reducing with time. For some flaps, particularly buried flap or bone and muscle only flaps, where it is not possible to assess the skin component, other methods of assessments are advocated such as implantable doppler probe. ${ }^{2}$

In this unit, the frequency of monitoring is hourly for the first 48 hours, four hourly for the next five days and then daily until time of discharge. Nursing staff monitor the flap on each occasion and record the findings on the flap chart. Unless called by the nursing staff for concern about the flap, junior doctors check the flap every 2 hours during the first 48 hours. On occasion this will result in an additional check if the hourly check has already taken place by the nurse. The four hourly or daily checks are undertaken by the junior doctor. 
The frequency of monitoring is a burden on both patient and staff. As the flap monitoring protocol was scheduled for review, it was felt that it would be useful to reflect on the patients' experience. A similar approach has been adopted for patient experience following tracheostomy, laser excision of tongue and the patient concerns inventory at diagnosis. $7,8,9$ Hence the aim of this study was to perform a cross-sectional survey of a consecutive group of patients following FTT to ascertain their experience of monitoring. To our knowledge, the incorporation of patient feedback has never been published before and this data could help refine practice.

\section{Patients and method}

The first stage of the study comprised discussions with current inpatients ready for discharge as well as those with previous microvascular reconstructive surgery at Aintree University Hospital. Patients were probed on their experiences before surgery, during and after flap monitoring. Based on their responses, a one page draft questionnaire was devised. The Aintree head and neck patient and carer support group further refined this into a final one page questionnaire with a total of 18 items, most having a 4-point Likert-type response option scale.

During the second stage of the study, a retrospective review was done over a 24 month period (2015 and 2016) for all patients who received flap monitoring for microvascular free flap under the maxillofacial department at Aintree University Hospital. Patients were identified using ORMIS theatre records. These patients were cross referenced on SIGMA for the 
inclusion criteria using clinical letters. Clinical details were collected from hospital records and included age, gender, type of flap site, tumour stage and site. Each patient was allocated a survey number to maintain anonymity. The questionnaire was sent by post and included a pre-paid return envelope in January 2017. A reminder was sent 3 weeks later. Patients known to be lost to follow up or who had died were excluded from the survey.

Fishers exact test (2 comparison groups) or Chi-squared test ( $>2$ groups) was used to test for associations between question responses and the clinical or demographic characteristics of the patient. For this purpose, responses to the first 11 questions were grouped into 'Not at all / A little' Vs 'Quite a bit / Very much'. Statistical significance was regarded as at the 5\% level. SPSS version 19 was used for the analyses. The study was approved by the Clinical Audit Department at Aintree University Hospital.

\section{Results}

A total of 178 consecutive patients underwent free-flap surgery between 1st January 2015 and 31st December 2016. At the time of survey (February 2017) 28 patients were known to have died. Of 150 questionnaires, two were returned uncompleted and analysable responses were received from 71\% (106/150). Response rates were slightly lower for younger patients (61\% if aged $<60$ years, $77 \%$ if $60-69$ years, $77 \%$ if 70 years or older), if $N$ staging was positive (59\%) rather than negative (77\%), if the free flap was hard composite (60\%) rather than soft (77\%) in nature and if the patient was ORN (58\%); otherwise response was less variable by sex, $\mathrm{T}$ stage, Tumour site and time from operation to survey (results not shown). The median (IQR) age of the sample responders was 67 (59-74) years and 52\% (55/106) were male. Almost three-quarters (72\%, 76) of free-flaps were soft (Radial forearm 51, ALT 22, ALT \& radial forearm 1, median sural artery perforator flap 2) and 28\% (30) were hard 
(fibula 11, scapula 7, DCIA 6, radial forearm 6). Seven patients were ORN, and of the others 46 were late staging T 3-4, and 24 were nodal positive; 29 had tumours located in the tongue, 20 as lower gum, 11 as buccal, 10 as floor of mouth with 29 others (including 12 maxilla and 7 soft palate). Median (IQR) time from operation to survey was 11 (5-15) months.

Overall sample results for questions 1 to 11 of the survey are shown in Table 1 . One quarter (28\%) could have received more information about flap monitoring. Expectations were largely met for $87 \%$ and most (84\%) felt reassured by the flap being checked. Patients were generally not worried that much by the flap observations (86\%) nor did they feel that monitoring stopped their recovery (93\%). The observations did however substantially disturb sleep for one-third (32\%) of patients. Just over half (53\%) were notably relieved when monitoring changed from hourly to every four hours, and almost all (98\%) expressed a lack of worry about this changeover. Nearly two-thirds (61\%) were particularly aware of having their flap checked 24 hours after surgery. Most (85\%) had few problems with pain during or after the assessment and almost all (93\%) were not especially distressed by the monitoring experience. Patients experiencing distress were asked to give further details and 5 mentioned problems with sleeping, 3 with a lack of understanding of what was going on or being checked, 1 in opening the mouth, 1 from bleeding from the corners of the mouth, 1 because the Doppler probe machine didn't seem to work properly and 1 who had to be taken back to theatre because of a complication.

In response to the rest of the questionnaire $12 \%(12 / 104)$ would have preferred less frequent observations during the day, and 45\% (47/105) would have preferred less frequent observations whilst asleep. Of those having a probe on the flap 89\% (31/35) found the probe reassuring. Most (60\%, 61/101) would have liked feedback after every observation. Patients 
were asked about nine potential problems and were asked to select any aspect they found most unpleasant, and these were: 'being woken from sleep' (55), 'opening mouth' (37), 'salivation' (29), 'touching flap' (14), 'pain' (13), 'coughing' (10) 'bright light' (7), 'difficulty breathing' (5), 'bleeding' (4),

There were very few associations at the $5 \%$ level $(\mathrm{P}<0.01)$ between categorical responses throughout the questionnaire and the clinical or demographic characteristics of the patient. Younger patients were more likely to be 'quite a bit' or 'very much' consciously aware of having their flap checked 24 hours following surgery (<60 years: 80\%, 24/30; 60-69 years: 64\%, 21/33; 70+ years; 45\% 19/42; $\mathrm{P}=0.01$ ). Males were less likely than females to be reassured by their flap probe (Males: 25\%, 4/16; Females: 0\%, 0/19; $\mathrm{P}=0.04$ ).

Patients were also asked about how the observations being done could have been improved and this their responses are summarised in Table 2. In addition, patients were also asked to add any further comments if they wished to and a summary of these are given in Table 3.

\section{Discussion}

To the best of our knowledge this survey is the first of its kind on the patients' perspective about flap monitoring. It was co-designed with the Patient and Carer Support Group at Aintree University Hospital. This allowed the items to be more patient centred in respect to their previous experiences, rather than the questionnaire written solely from the view-point of the clinician.

A consecutive series of patients were asked to participate in the survey and the number and response rate of $71 \%$ was satisfactory. The time from operation to survey varied between 
patients with most having surgery within 18 months, the longest being 23 months. Memory recall could possibly have had an effect on their responses. We recognize the limitations of the retrospective design where it might be difficult for patients to recall their true experience and a prospective study would give more contemporaneous reflection. With an average of two flaps a week, such a design would take several years to complete.

The survey has shown that about a quarter of patients felt they did not receive enough information about flap monitoring. The amount of information that patients and their carers wish for before treatment is very variable (4), however it would seem reasonable to produce a free flap monitoring specific patient information leaflet and have that accessible to patients in their information pack before surgery and on-line. This leaflet would explain the rational for monitoring and what is involved, and could also include the findings from this survey in terms of patient experience.

Most patients felt reassured by the flap monitoring but they would like more feedback. In the initial 24 hours the frequency of monitoring did not seem such an issue with 39\% of patients either 'a little' or 'not at all' aware of having their free flap monitored. This is probably in part due to all the other intensive monitoring taking place in this immediate time period after the operation.

Although patients felt that monitoring did not stop their recovery, the impact on sleep was repeatedly highlighted throughout the survey. There was relief when the flap monitoring changed to four hourly though the reason for this is unclear. It might reflect a confidence that the flap has been successful rather than relief that the checks are becoming less intrusive. Patients were not worried about the change in frequency and this probably reflects confidence 
in the clinical team. Most patients were not distressed by monitoring. Patients with an implantable doppler found this reassuring though the numbers were small $(n=35)$. Ho et al published a rationale for the use of the implantable Doppler probe based on 7 years experience and emphasised that implantable Doppler probe had its place in selected cases. ${ }^{10}$

The main finding of this study is that, not surprisingly, flap monitoring is associated with interruption of sleep. As long as it is safe to do so, patients would prefer less frequent monitoring overnight. As an outcome of this survey consideration is being given to reducing the frequency of monitoring to two hourly from midnight to 6 am on day two hence omitting a flap check at $1 \mathrm{am}, 3$ am and $5 \mathrm{am}$. This minor change could make a substantial difference to a patient but the impact of this change on flap salvage and failure needs to be audited. During the day following the first 24 hours, the flap monitoring reverts back to hourly until day three where it changes to four hourly, as per current protocol. Discussions are taking place as to whether it would be appropriate to alter the night observations to 2 hourly on day two and 6 hourly on day 3-5. It is hoped that patients might appreciate better sleep and the impact of the change in protocol will be monitored in terms of flap salvage and success and patient feedback.

\section{Conflict of interest statement}

We have no conflicts of interest.

\section{Ethics statement/confirmation of patient's permission}

The data, which had been collected as part of a service audit rather than for research, met the criteria of the local Clinical Governance Department for service evaluation. 


\section{Acknowledgements}

We would like to that the Head and Neck Cancer Patient and Carer Research Forum based at the Regional Surgical Centre, for helping to develop and pilot the study questionnaire.

\section{References}

1. Abdel-Galil K, Mitchell D. Postoperative monitoring of microsurgical free tissue transfers for head and neck reconstruction: a systematic review of current techniques--part I. Non-invasive techniques. Br J Oral Maxillofac Surg. 2009 Jul;47(5):351-5

2. Abdel-Galil K, Mitchell D. Postoperative monitoring of microsurgical free tissue transfers for head and neck reconstruction: a systematic review of current techniques--part II. invasive techniques. Br J Oral Maxillofac Surg. 2009 Jul;47(6):438-42

3. J. S. Brown, J. C. Devine, P. Magennis, P. Sillifant, S. N. Rogers, E. D. Vaughan Factors that influence the outcome of salvage in free tissue transfer, $\mathrm{Br} \mathrm{J}$ Oral Maxillofac Surg. 2003 Feb;41(1):16-20.

4. Chen KT, Mardini S, Chuang DC, et al. Timing of presentation of the first signs of vascular compromise dictates the salvage outcome of free flap transfers. Plast Reconstr Surg. 2008 Sep;122(3):991-2

5. Patel UA, Hernandez D, Shnayder Y et al. Free Flap Reconstruction Monitoring Techniques and Frequency in the Era of Restricted Resident Work Hours. JAMA Otolaryngol Head Neck Surg. 2017 Jun 1 
6. I.S. Whittaker, V. Gulati, G.L. Ross, A. Menon, T.K. Ong, Variations in the postoperative management of free tissue transfers to the head and neck in the United Kingdom Br J Oral Maxillofac Surg. 2007 Jan;45(1):16-8.

7. Rogers SN, Russell L, Lowe D. Patients' experience of temporary tracheostomy after microvascular reconstruction for cancer of the head and neck. Br J Oral Maxillofac Surg. 2017 Jan;55(1):10-1

8. Gilmartin M, Ali T, Rogers SN. Patients' Experience in the Early Recovery Phase After Removal of Intraoral Squamous Cell Carcinoma With Carbon Dioxide Laser. Br J Oral Maxillofac Surg. 2017 May;55(4):388-390

9. Byrne M, Rogers SN Audit of Patients views on the Patients Concerns Inventory (at diagnosis). British Journal of Oral and maxillofacial Surgery (2107), in Press.

10. Ho MW, Cassidy C, Brown JS, Shaw RJ, Bekiroglu F, Rogers SN. Rationale for the use of the implantable Doppler probe based on 7 years' experience. $\mathrm{Br} \mathrm{J}$ Oral Maxillofac Surg. 2014 Jul;52(6):530-4 
Table 1. Patient response to the first 11 questions of the survey

\begin{tabular}{|c|c|c|c|c|c|c|}
\hline & Not at all & A little & Quite a bit & $\begin{array}{l}\text { Very } \\
\text { much }\end{array}$ & $\begin{array}{c}\% \text { not at } \\
\text { all / A } \\
\text { little }\end{array}$ & $\begin{array}{c}\text { \% Quite a } \\
\text { bit/ Very } \\
\text { much }\end{array}$ \\
\hline 1. Did you receive enough information about flap monitoring? & 6 & 24 & 37 & 39 & $28 \%$ & $72 \%$ \\
\hline 2. Did the flap monitoring meet your expectations? & 3 & 10 & 30 & 59 & $13 \%$ & $87 \%$ \\
\hline 3. Did you feel reassured by the flap being checked? & 1 & 5 & 18 & 81 & $6 \%$ & $84 \%$ \\
\hline 4. Did the flap observations worry you? & 64 & 27 & 8 & 7 & $86 \%$ & $14 \%$ \\
\hline 5. Did the monitoring stop your recovery? & 92 & 6 & 1 & 6 & $93 \%$ & $7 \%$ \\
\hline 6. Did the observations disturb your sleep? & 19 & 52 & 17 & 17 & $68 \%$ & $32 \%$ \\
\hline $\begin{array}{l}\text { 7. Were you consciously aware that you were having your flap } \\
\text { checked } 24 \text { hours following surgery? }\end{array}$ & 16 & 25 & 16 & 48 & $39 \%$ & $61 \%$ \\
\hline 8. Did you experience pain during/after the assessment? & 70 & 19 & 8 & 8 & $85 \%$ & $15 \%$ \\
\hline $\begin{array}{l}\text { 9. Were you relieved when monitoring changed to every four hours } \\
\text { after } 48 \text { hours of hourly observations? }\end{array}$ & 19 & 30 & 30 & 25 & $47 \%$ & $53 \%$ \\
\hline 10. Were you worried when this monitoring period changed? & 79 & 22 & 1 & 1 & $98 \%$ & $2 \%$ \\
\hline 11. Did you find the monitoring distressing? & 81 & 11 & 1 & 6 & $93 \%$ & $7 \%$ \\
\hline
\end{tabular}


Table 2. A summary of patients comments (verbatim) as to how the observations could have been improved

- All the doctors carrying out the observations

- All very efficient

- By providing more information prior to, during and after

- can't see a way around it, necessary evil.

Cannot be improved.

- Excellent treatment by all levels of staff

- I couldn't I feel I was treated brilliantly

- Idon't know

- I don't think it can be improved, checking is necessary

- I don't think it could be improved without compromising health of the patient

- I was very happy with standard of checks + reassured by team

- In my opinion- the observations are crucial

- In my personal opinion and experience I found the monitoring of the flap no problem, as it reassured me.

- Less frequent after first 24 hours

- Less frequent in early hours

- Less people around the bed

- More info on flap healing even if all okay to reassure.

- No suggestions

- One stitch broke which was distressing because of the bleeding and possible failure of flap.

- Reduced number of times woken overnight

- $\quad$ Since monitoring is necessary it is for the professional to decide

- Stress how well patient is doing

- Unless life threatening let people sleep, it's hard enough in hospital

- Wi-fi probe to doctors office 


\section{Table 3. A summary of patients further comments (verbatim)}

- Although a very unpleasant procedure to have done I could not praise staff (medics/nurses) for kind care and attention and have since made excellent progress. The most unpleasant part was the stay on intensive care unit due to sleep deprivation. In fact sleep deprivation was possibly the worse part of the experience

- $\quad$ Although being woken up is unpleasant I fully understand how important it is to be monitored. The care I received throughout my stay was excellent and the first 28 hours on the critical care unit was superb- everything was dealt with in a calm and caring way and all staff kept disturbances to a minimum. I am extremely grateful for the care I received

- Although I have indicated in response to Q13 that being woken from sleep was the worst aspect of being monitored, in reality I was rarely asleep anyway; during the first few days I found it very hard to sleep when the trachy was in place, and as a "side sleeper" lying in my natural position was difficult because of the restrictions of the tube. In fact, the night time checks were a welcome spot of punctuation, serving to break up the long hours of sleeplessness! In Addition, I was aware of the critical nature of the first 48 hours in relation to the success of otherwise of the flap, so felt that any minor discomfort or inconvenience afforded by the frequent monitoring was more than outweighed by the reassurance it provided.

- Care very professional and efficient. Only found the monitoring distressing for the first 24 hours. Just felt the need to sleep and be left alone. However did realise the monitoring was for my benefit and appreciated the excellent care I was given. All staff were very caring.

- Everything was brilliant, very professional. The people (staff) lovely people. The way a hospital should run. Well done, keep it up!

- $\quad$ Found the flap monitoring very reassuring and increased my confidence and made me less anxious. Thank you

- I actively found the checks to be reassuring and all the staff who carried out the checks were so pleasant (and apologetic) that it wasn't really a problem. Compared to the other issues I had to deal with at the time, I suppose this was bottom of the list. My personal view is that if something is going wrong the sooner it is diagnosed the better.

- I am sorry I can't fill this survey in as I was in ICU for 6 days. Don't remember much about it. All I can say I got the best of care in ICU and on ward 28 thank you

- I found the monitoring very reassuring, particularly the comments of the young doctors who always mentioned the nice pink colour of the flap and that everything was fine! During this monitoring I realise that some doctors were assigned to do the monitoring for a period (probably a shift). I thought this was great as it enabled a bond of trust to grow between the doctor and patient. The encouragement given by all the doctors and nursing staff was wonderful and so important. I could not have asked for better. thank you so much.

- I had two spells of infection and in total spent 38 days at Aintree so was able to judge over an extended period, I feel my treatment was completely satisfactory and can recommend Aintree Hospital to anybody

- I really can't remember a lot about my cancer op. But if you call me I would be glad to help.

- I was always shown the highest degree of respect and caring. Thank you. The Macmillan nurses who took care of me during my stay at Aintree were dedicated to all patients. They ere faultless. Thank you. My flap monitoring was checked more frequently. I did have feedback the doctors/staff were faultless and informed every time they examined me of my progress. thank you.

- I was told it was ok after each check

- I would like to say the team that look after me where very professional and treated me with the utmost courtesy and were very friendly

- I would sooner had all the checking to make sure it took. That's the main thing. I would like to thank everyone for the help.

- In my opinion they were done to my expectations and feedback was given professionally

- Once I was fully aware + more awake, the persons examining my mouth told me everything looked fine and this was very comforting

- Thanks to all the team involved in my procedure a little discomfort is a small price to pay.

- The care and after care whilst in hospital, in my opinion was "absolutely fantastic" thank you all so very much

- The night observations didn't bother me too much as I wasn't sleeping very well

- The way the observations were conducted did not bother me in any way

- Were considerate, would wake with a gentle touch (although not needed a lot of the time) and were encouraging in their comments. I thought OBS were every 2 hours after 24 hours. I found the tracheostomy more challenging but was told that up front. 